\title{
A GENERALIZATION OF THE TRANSFER MAP IN THE COHOMOLOGY OF GROUPS $\left({ }^{1}\right)$
}

BY

\section{LEONARD EVENS}

1. Introduction. In this paper a multiplicative object analogous to transfer is defined and its properties discussed. The original motivation for defining such an object was to give relatively precise information about nontriviality for restriction and related questions as discussed by Swan [7] and the author [4, §7]. In addition we find ourselves led to interesting formulas relating this object to the Chern classes of representations as defined by Atiyah [1] and others. This relation will be the subject of a future paper.

2. Wreath products and the monomial representation. Let $G$ be a group and $H$ a subgroup of finite index $l$. Then there is a natural construction (going back to Frobenius) whereby $G$ may be imbedded in the wreath product of the symmetric group $\mathscr{S}_{l}$ with $H$. This is described below.

We shall use the notation of Hall $[5, \S 5.9]$ concerning wreath products except that for us permutations will compose as functions and act on the left. Thus if $S_{i}$ is a permutation group on the set $L_{i}=\left\{1,2, \cdots, l_{i}\right\}(i=1,2)$, then $S_{1} \int S_{2}$ is the group of permutations $\theta$ on $L_{1} \times L_{2}$ satisfying

$$
\theta(i, j)=\left(a i, \tau_{i} j\right)
$$

where

$$
\sigma \in S_{1}, \tau_{i} \in S_{2} \quad\left(i=1,2, \cdots, l_{1}\right) .
$$

Also, ordering $L_{1} \times L_{2}$ lexicographically, we may identify it with $L=\left\{1,2, \cdots, l_{1} l_{2}\right\}$ so that $S_{1} \int S_{2}$ may be viewed as a subgroup of $\mathscr{S}_{l_{1} l_{2}}$.

If $S$ is a permutation group of degree $l$ and $H$ is an abstract group then we have $S \int H$, a permutation group on $L \times H$ where $H$ acts on itself by left translation. In fact $S \int H$ is the semi-direct product of $S$ with $H^{l}$ subject to

$$
\pi^{-1} \sigma_{1} \times \sigma_{2} \times \cdots \times \sigma_{l} \pi=\sigma_{\pi(1)} \times \sigma_{\pi(2)} \times \cdots \times \sigma_{\pi(l)}
$$

for $\pi \in S$ and $\sigma_{i} \in H$.

Received by the editors March 10, 1962.

(1) Work done with partial support from National Science Foundation Grants 9504 and 19137. 
Let $\tau_{1}, \tau_{2}, \cdots, \tau_{l}$ be a left transversal of $H$ in $G$. For $\rho \in G$ we have

$$
\rho \tau_{i}=\tau_{\pi(i)} \sigma_{i}
$$

for uniquely determined $\pi \in \mathscr{S}_{l}, \sigma_{i} \in H$.

Define

$$
\Phi(\rho)=\pi \cdot \sigma_{1} \times \sigma_{2} \times \cdots \times \sigma_{l} .
$$

Then $\Phi: G \rightarrow \mathscr{S}_{l} \int H$ is a monomorphism.

If $\tau_{1}^{\prime}, \tau_{2}^{\prime}, \cdots, \tau_{l}^{\prime}$ is another transversal,

$$
\tau_{i}^{\prime}=\tau_{\alpha(i)} \beta_{l}
$$

where $\alpha \in \mathscr{S}_{l}$ and $\beta_{i} \in H$. Letting

$$
\mu=\alpha \cdot \beta_{1} \times \beta_{2} \times \cdots \times \beta_{l}
$$

we see that $\Phi^{\prime}$ defined from the second transversal is related to $\Phi$ by

$$
\Phi^{\prime}=C_{\mu} \circ \Phi .
$$

(Here $C_{\mu}(\gamma)=\mu^{-1} \gamma \mu$ for $\mu \in \mathscr{S}_{l} \int H$.)

3. The monomial module. Let $A$ be a left $H$-module; we construct a corresponding $\mathscr{S}_{l} \int H$-module as follows. Set

$$
A^{l}=A \otimes_{z} A \otimes_{z} \cdots \otimes_{z} A \text { ( } l \text { times) }
$$

and define the operation of $\mathscr{S}_{l} \int H$ on this group by the formulas

$$
\begin{aligned}
\left(\sigma_{1} \times \sigma_{2} \times \cdots \times \sigma_{l}\right)\left(a_{1} \otimes \cdots \otimes a_{l}\right) & =\sigma_{1} a_{1} \otimes \sigma_{2} a_{2} \otimes \cdots \otimes \sigma_{l} a_{l}, \\
\pi\left(a_{1} \otimes a_{2} \otimes \cdots \otimes a_{l}\right) & =a_{\pi^{-1}(1)} \otimes a_{\pi^{-1}(2)} \otimes \cdots \otimes a_{\pi^{-1}(l)},
\end{aligned}
$$

for $\sigma_{l} \in H$ and $\pi \in \mathscr{S}_{l}$. More generally if $C$ is an $H$-complex in the sense of [3], then by introducing the appropriate sign $C^{l}$ is made an $\mathscr{S}_{l} \int H$-complex. (See $[4, \S 5$, p. 231].)

Suppose now that $G$ is a group containing $H$ as a subgroup of finite index $l$. By means of the injection $\Phi$ we may view $A^{l}$ as a $G$-module which we call the monomial $G$-module induced from the $H$-module $A$ and denote by $\mathscr{M}_{H \rightarrow G}(A)$. Naturally this definition depends on the choice of coset representatives. If $\tau_{1}^{\prime}, \tau_{2}^{\prime}, \cdots, \tau_{l}^{\prime}$ is another transversal, it yields another injection $\Phi^{\prime}$ and another structure $\mathscr{M}^{\prime}$ of $G$-module on $A^{l}$. Then in fact the action of the element $\mu$ (formula (1)) on $A^{l}$ yields a mapping

$$
\mu: \mathscr{M}^{\prime} \rightarrow \mathscr{M}
$$

which is an isomorphism of $G$-modules.

As an example let $A=Z \oplus B$ where $B$ is an $H$-module. Then

$$
\mathscr{M}_{H \rightarrow G}(A)=\mathscr{M}_{0} \oplus \mathscr{M}_{1} \oplus \cdots \oplus \mathscr{M}_{l}
$$

where $\mathscr{M}_{0} \cong Z, \mathscr{M}_{1} \cong Z(G) \otimes_{H} B$ (the induced module), and $\mathscr{M}_{l} \cong \mathscr{M}_{H \rightarrow G}(B)$. 
4. The norm map. Preliminaries on wreath products. In this section we outline the properties of a special case of the wreath product of cohomology classes which has been defined generally by Nakaoka.

Suppose first that $C$ is a cochain complex (i.e., a complex involving only nonnegative degrees). As in $\S 3$ make $C^{l}$ an $\mathscr{S}_{l}$-complex.

Let $S$ be a subgroup of $\mathscr{S}_{l}$ and let $W$ be an $S$-projective resolution of $Z$. We wish to study the double complex $D=\operatorname{Hom}_{S}\left(W, C^{l}\right)\left({ }^{2}\right)$. The homology of $D$ (and also its other invariants [3, Chapter XV, §6]) is independent of the particular projective resolution $W$ chosen. Also if $F: C \rightarrow C^{\prime}$ is a map of complexes of degree zero then $F$ induces

$$
\operatorname{Hom}_{s}\left(1, F^{l}\right): \operatorname{Hom}_{s}\left(W, C^{l}\right) \rightarrow \operatorname{Hom}_{S}\left(W, C^{\prime l}\right),
$$

a map of double complexes. By a lemma of Steenrod [6, Lemma 3.2], homotopic maps of $C$ into $C^{\prime}$ induce homotopic (but not bi-homotopic) maps of the corresponding (double) complexes. Thus the homology of $\operatorname{Hom}_{S}\left(W, C^{l}\right)$ depends only on the homotopy type of the complex $C$.

Following Borel and Hirzebruch [2], denote by $H^{* *}(C)$ the direct product of the groups $H^{n}(C)$. (Then if $C$ is also a differential graded ring, $H^{* *}(C)$ has a a resulting ring structure.)

Consider in particular the complex $Z[T]$ ( $T$ an indeterminate) where the grading is by degree and the differentiation is trivial. Then $C^{l}$ may be identified with the polynomial ring $Z\left[T_{1}, T_{2}, \cdots, T_{l}\right]$ on which $\mathscr{S}_{l}$ acts by permuting the variables and multiplying by the appropriate sign. (Let $T_{1}=T \otimes 1 \otimes \cdots \otimes 1$, etc.). Write

Then

$$
D_{0}=\operatorname{Hom}_{S}\left(W, Z\left[T_{1}, T_{2}, \cdots, T_{l}\right]\right) .
$$

$$
H^{*}\left(D_{0}\right) \cong H^{*}\left(S, Z\left[T_{1}, \cdots, T_{l}\right]\right)
$$

and

$$
H^{* *}\left(D_{0}\right) \cong H^{* *}\left(S, Z\left[\left[T_{1}, T_{2}, \cdots, T_{l}\right]\right]\right) .
$$

(Here we use the fact that $W$ may be chosen so that each $W_{p}$ is finitely generated.)

Now suppose $\alpha \in H_{e}^{* *}(C)=\prod_{n \geqq 0} H^{2 n}(C)$. (Generally we shall write such elements as infinite series $\alpha=\alpha_{0}+\alpha_{1}+\cdots+\alpha_{n}+\cdots$ with $\alpha_{n} \in H^{2 n}(C)$.) Let $a=a_{0}+a_{1}+\cdots+a_{n}+\cdots$ be a cocycle representing $\alpha$. Then we may define a map

$$
F: Z[T] \rightarrow C
$$

of complexes such that $F\left(T^{2 n}\right)=a_{n}, F\left(T^{2 n+1}\right)=0$, and

$$
F^{* *}\left(1 /\left(1-T^{2}\right)\right)=\alpha \text {. }
$$

Moreover, if $a^{\prime}$ also represents $\alpha$ and $F^{\prime}$ is defined for $a^{\prime}$, then $F$ and $F^{\prime}$ are homotopic. Let

(2) Following [3], $\operatorname{Hom}(X, Y)=\Sigma_{p, q} \operatorname{Hom}\left(X_{p}, Y^{q}\right)$ with the obvious differentiations. 


$$
Y=\prod_{i=1}^{l}\left(1 /\left(1-T_{i}^{2}\right)\right) .
$$

$Y$ is an element of $Z\left[\left[T_{1}, \cdots, T_{l}\right]\right]^{S}$ which is naturally isomorphic to

$$
H^{0}\left(S, Z\left[\left[T_{1}, \cdots, T_{l}\right]\right]\right) \text {. }
$$

Define $1_{S} \int \alpha$ in $H_{e}^{* *}\left(\operatorname{Hom}_{S}\left(W, C^{l}\right)\right)$ by the formula

$$
1_{s} \int \alpha=\operatorname{Hom}_{S}\left(1, F^{l}\right)^{* *}(Y) \text {. }
$$

In view of the above remarks, this object is well defined, it does not depend on $a, F$, or even $W$.

We now summarize certain properties of the operation of forming $1 \int \alpha$ which will be useful below.

1. Let $S^{\prime}<S$ and suppose $G: C \rightarrow C^{\prime}$ is a map of complexes. Then there an induced map

$$
G^{* *}: H^{* *}\left(\operatorname{Hom}_{S}\left(W, C^{l}\right)\right) \rightarrow H^{* *}\left(\operatorname{Hom}_{S^{\prime}}\left(W, C^{\prime l}\right)\right) .
$$

(In fact, we could replace $W$ by any $S^{\prime}$-projective resolution of $Z$ in the second term.) Then

$$
G^{* *}\left(1_{S} \int \alpha\right)=1_{S^{\prime}} \int G^{* *}(\alpha)
$$

2. Let $S_{1}<\mathscr{S}_{l_{1}}, S_{2}<\mathscr{S}_{l_{2}}$ and let $W_{1}$ and $W_{2}$ be corresponding projective resolutions of $Z$. As in [4, §5, p. 231], $W_{1} \otimes W_{2}^{l_{2}}=W$ is an $S_{1} \int S_{2}=S$-projective resolution of $Z$. In the obvious way define the homomorphism (of functors) $\left({ }^{3}\right.$ ).

$$
\mathscr{A}: \operatorname{Hom}_{S_{1}}\left(W_{1}, \operatorname{Hom}_{S_{2}}\left(W_{2}, C^{l_{2}}\right)^{l_{1}}\right) \rightarrow \operatorname{Hom}_{S_{1} \int S_{2}}\left(W, C^{l_{1} l_{2}}\right) .
$$

(Here $S_{1} \int S_{2}$ is embedded in $\mathscr{S}_{l_{1} l_{2}}$ as in $\$ 1$.) Now for $\alpha \in H^{* *}(C), 1_{S_{1}} \int\left(1_{S_{2}} \int \alpha\right)$ makes sense as an element of the homology of the left-hand side and

$$
\mathscr{A}^{* *}\left(1_{S_{1}} \int\left(1_{S_{2}} \int \alpha\right)\right)=1_{S_{1} \int s_{2}} \int \alpha .
$$

3. Let $S_{1}$ and $S_{2}$ be as above and let $l=l_{1}+l_{2}$. Then $S_{1} \times S_{2}$ may be imbedded in $\mathscr{S}_{l}$ by letting $S_{1}$ act on the first $l_{1}$ symbols and $S_{2}$ on the remaining $l_{2}$ symbols. Also $W_{1} \otimes W_{2}$ is an $S_{1} \times S_{2}$-projective resolution of Z. Again consider the obvious homomorphism $\left({ }^{3}\right)$

$$
\mathscr{B}: \operatorname{Hom}_{S_{1}}\left(W_{1}, C^{l_{1}}\right) \otimes \operatorname{Hom}_{S_{2}}\left(W_{2}, C^{l_{2}}\right) \rightarrow \operatorname{Hom}_{S_{1} \times S_{2}}\left(W_{1} \otimes W_{2}, C^{l}\right) .
$$

Now the external product $1_{S_{1}} \int \alpha \times 1_{S_{2}} \int \alpha$ is an element of the homology of the left-hand side and

$$
\mathscr{B}^{* *}\left(1_{S_{1}} \int \alpha \times 1_{S_{2}} \int \alpha\right)=1_{S_{1} \times S_{2}} \int \alpha .
$$

(3) Here since $C$ is a complex we must include a sign change in the definitions of $\mathscr{A}$ and $\mathscr{B}$. 
4. Let $S<\mathscr{S}_{l}$, let $W$ be an $S$-projective resolution of $Z$ and $\Delta: W \rightarrow W \otimes W$ a diagonal map. Let $C_{1}$ and $C_{2}$ be cochain complexes. Then $\Delta$ (and permuting the factors of the tensor product) induces a homomorphism

$$
\operatorname{Hom}_{S \times s}\left(W \otimes W, C_{1}^{l} \otimes C_{2}^{l}\right) \rightarrow \operatorname{Hom}_{s}\left(W,\left(C_{1} \otimes C_{2}\right)^{l}\right) .
$$

(Here $S \times S$ acts on $C_{1}^{l} \otimes C_{2}^{l}$ "componentwise" and $S$ acts on $\left(C_{1} \otimes C_{2}\right)^{l}$ as usual.) Combining this homomorphism with one defined as in (3) we get a homomorphism

$$
\mathscr{P}: \operatorname{Hom}_{S}\left(W, C_{1}^{l}\right) \otimes \operatorname{Hom}_{S}\left(W, C_{2}^{l}\right) \rightarrow \operatorname{Hom}_{S}\left(W,\left(C_{1} \otimes C_{2}\right)^{l}\right) .
$$

Let $\alpha_{i} \in H_{e}^{* *}\left(C_{i}\right)(i=1,2)$. Then $1_{S} \int \alpha_{1} \times 1_{s} \int \alpha_{2}$ is in the homology of the lefthand side, $\alpha_{1} \times \alpha_{2} \in H_{e}^{* *}\left(C_{1} \otimes C_{2}\right)$, and

$$
\mathscr{P}^{* *}\left(1_{S} \int \alpha_{1} \times 1_{s} \int \alpha_{2}\right)=1_{S} \int \alpha_{1} \times \alpha_{2} .
$$

If in addition $C_{1}=C_{2}=C$ and $C$ is a differential graded ring, then using $\mathscr{P}$, we can make $\operatorname{Hom}_{S}\left(W, C^{l}\right)$ such a ring and

$$
\left(1_{s} \int \alpha_{1}\right)\left(1_{s} \int \alpha_{2}\right)=1_{s} \int \alpha_{1} \alpha_{2}
$$

5. To verify the above statements or generally to study $1_{s} \int \alpha$ it is convenient to reduce things to the cochain level. Let $a$ represent $\alpha \in H_{e}^{* *}(C)$ as above. Let $\varepsilon: W \rightarrow Z$ be the augmentation for the resolution $W$. Define the cocycle $\varepsilon \int a \in \operatorname{Hom}_{S}\left(W, C^{l}\right)$ by

$$
\left(\varepsilon \int a\right)(w)=\varepsilon(w)(a \otimes a \otimes \cdots \otimes a) .
$$

Then $\varepsilon \int a$ represents $1_{s} \int \alpha$.

5. The norm map. Let $H$ be a group and suppose $S<\mathscr{S}_{l}$, etc., as above. Take $C=\operatorname{Hom}_{H}(X, A)$ where $X$ is an $H$-projective resolution of $Z$ and $A$ is an $H$-module. Then $H^{* *}(C)=H^{* *}(H, A)$ and we wish to extend the definition of $\$ 4$ so as to define an element $1_{S} \int \alpha$ (using the same notation) in $H^{* *}\left(S \int H, A^{l}\right)$. As in $\$ 4$, we have the homomorphism

$$
\mathscr{A}_{1}: \operatorname{Hom}_{S}\left(W, \operatorname{Hom}_{H}(X, A)^{l}\right) \rightarrow \operatorname{Hom}_{S} \int_{H}\left(W \otimes X^{l}, A^{l}\right) .
$$

Define $1_{s} \int \alpha$ (for $\alpha \in H_{e}^{* *}(H, A)$ ) in the homology of the right-hand side as $\mathscr{A}_{1}^{* *}\left(1_{S} \int \alpha\right)$. Since $\mathscr{A}_{1}$ is a homomorphism even of functors the definition is well made. The facts outlined in $\$ 4$ carry over as follows:

1. If $\phi: H^{\prime} \rightarrow H$ is a homomorphism of groups, $f: A \rightarrow A^{\prime}$ a consistent homomorphism of $H$-modules we have $\left(i: S^{\prime}<S\right.$ )

$$
\left(i \int \phi\right)^{* *}\left(1_{S^{\prime}} \int \alpha\right)=1_{S^{\prime}} \int \phi^{* *}(\alpha)
$$


2. We have an isomorphism of groups

and

$$
\mathscr{A}_{2}:\left(S_{1} \int S_{2}\right) \int H \stackrel{\cong}{\rightarrow} S_{1}\left(S_{2} \int H\right)
$$

$$
\mathscr{A}_{2}^{* *}\left(1_{s_{1}} \int\left(1_{s_{2}} \int \alpha\right)\right)=1_{s_{1}} \int s_{2} \int \alpha .
$$

3. We have an isomorphism

and

$$
\mathscr{B}_{2}: S_{1} \times S_{2} \int H \cong S_{1} \int H \times S_{2} \int H
$$

$$
\mathscr{B}_{2}^{* *}\left(1_{S_{1}} \int \alpha \times 1_{S_{2}} \int \alpha\right)=1_{S_{1} \times S_{2}} \int \alpha .
$$

4. Let $H_{1}$ and $H_{2}$ be groups and $A_{1}, A_{2}$ corresponding modules over $H_{1}, H_{2}$. The diagonal monomorphism $S \rightarrow S \times S$ induces a monomorphism

$$
\mathscr{P}_{2}: S \int H_{1} \times H_{2} \rightarrow S \int H_{1} \times S \int H_{2}
$$

$\mathscr{P}_{2}$ induces

and

$$
\mathscr{P}_{2}^{* *}: H^{* *}\left(S \int H_{1} \times S \int H_{2}, A_{1}^{l} \otimes A_{2}^{l}\right) \rightarrow H^{* *}\left(S \int H_{1} \times H_{2},\left(A_{1} \otimes A_{2}\right)^{l}\right)
$$

$$
\mathscr{P}_{2}^{* *}\left(1_{s} \int \alpha_{1} \times 1_{s} \int \alpha_{2}\right)=1_{s} \int \alpha_{1} \times \alpha_{2} .
$$

If $H_{1}=H_{2}=H$ and $A_{1} \otimes A_{2} \rightarrow A_{3}$ is an $H$ pairing, then there is a corresponding $S \int H$ pairing $A_{1}^{l} \otimes A_{2}^{l} \rightarrow A_{3}^{l}$ and using the cup product with respect to these pairings we have

$$
1_{s} \int \alpha_{1} \cup 1_{s} \int i \alpha_{2}=1_{s} \int \alpha_{1} \cup \alpha_{2} .
$$

In case $A_{1}=A_{2}=A_{3}=k$ is an $H$-ring (with the product pairing), formula (2) applies. In addition if $k$ is commutative and $H$ acts trivially the product mapping $k^{l} \rightarrow k$ is a homomorphism of $S \int H$ rings (where $S \int H$ acts trivially on $k$ ) and we may use this map to carry $1_{s} \int \alpha$ to an element $H_{l}^{* *}\left(S \int H, k\right)$. Using the same notation for this new element, we see that formula (2) still applies (and in fact all the above formulas).

5. If $a \in \operatorname{Hom}_{H}(X, A)$ represents $\alpha$, then $\varepsilon \otimes a^{l} \in \operatorname{Hom}_{S \int_{H}}\left(W \otimes X^{l}, A^{l}\right)$ represents $1_{s} \int \alpha$.

Let $G$ be a group, $H$ a subgroup of finite index $l$. Let $\Phi: G \rightarrow \mathscr{S}_{l} \int H$ be defined with respect to some transversal. For $\alpha \in H_{e}^{* *}(H, A)$ we define $\mathscr{N}_{H \rightarrow G}(\alpha) \in H_{e}^{* *}\left(G, \mathscr{M}_{H \rightarrow G}(A)\right)$ by

$$
\mathscr{N}_{H \rightarrow G}(\alpha)=\Phi^{* *}\left(1_{\mathscr{S}_{l}} \int \alpha\right)
$$

Notice that $\mathscr{S}_{l}$ may be replaced by the transitive subgroup $S$ which is the image of the composite homomorphism

$$
G \rightarrow \mathscr{S}_{l} \int H \stackrel{\text { surjection }}{\longrightarrow} \mathscr{S}_{l} .
$$


(This is the subgroup of permutations of the coset space $G / H$ produced by the action of $G$.)

If $\Phi^{\prime}: G \rightarrow \mathscr{S}_{l} \int H$ is defined with respect to another transversal and $\mathscr{N}_{H \rightarrow G}^{\prime}$ is defined by $\Phi^{\prime}$, then

$$
\mathscr{N}_{H \rightarrow G}(\alpha)=\mu^{* *} \mathscr{N}_{H \rightarrow G}^{\prime}(\alpha)
$$

Thus $\mathscr{N}_{H \rightarrow G}(\alpha)$ is essentially well defined.

If $k$ is a commutative ring on which $G$ acts, then $\mathscr{M}_{H \rightarrow G}(k)$ becomes a $G$-ring (treating $k$ as an $H$-ring), and $\psi: \mathscr{M}_{H \rightarrow G}(k) \rightarrow k$ defined by

$$
\psi\left(a_{1} \otimes a_{2} \otimes \cdots \otimes a_{l}\right)=\tau_{1} a_{1} \cdot \tau_{2} a_{2} \cdots \cdot \tau_{l} a_{l}
$$

(where $\tau_{1}, \cdots, \tau_{l}$ is the same transversal as that in the definition of $\mathscr{M}_{H \rightarrow G}(k)$ ) is a homomorphism of $G$-rings.

Given $\alpha \in H_{e}^{* *}(H, k)$, define $\mathscr{N}_{H \rightarrow G}(\alpha) \in H_{e}^{* *}(G, k)$ as $\psi^{* *} \mathscr{N}_{H \rightarrow G}(\alpha)$ (again using the same notation). With coefficients in $k$ there is no ambiguity in the module and no ambiguity in the definition of $\mathscr{N}_{H \rightarrow G}$.

REMARK. Notice the fact that since the group $G$ is smaller than $\mathscr{S}_{l} \int H$, it is possible to drop the restriction of trivial action required in the more general situation.

6. Formal properties. In what follows it will be possible to interpret the formal rules in different contexts depending on whether the module is general or a commutative ring on which the group $G$ acts. The contention always will be that the formula in question is valid in all such contexts.

Since the module $\mathscr{M}_{H \rightarrow G}(A)$ is not well defined except up to isomorphism there will be some ambiguity for the general situation. We shall ignore this ambiguity and write " =" where there is only a canonical isomorphism derived from an element $\mu$ as in $\S \S 2$ and 3 . We can always insure true equality by choosing transversals consistently.

Finally we assume always that $\alpha \in H_{e}^{* *}(H, A)$, etc.

1. Transitivity. Let $G>K>H$ where $H$ is of finite index $l$. Let $l_{1}=(G: K)$, $l_{2}=(K: H)$ so that $l=l_{1} l_{2}$. Let $\tau_{1}^{\prime}, \tau_{2}^{\prime}, \cdots, \tau_{l_{1}}^{\prime}$ be a transversal of $K$ in $\dot{G}$, $\tau_{1}^{\prime \prime}, \tau_{2}^{\prime \prime}, \cdots, \tau_{l_{2}}^{\prime \prime}$ a transversal of $H$ in $K$. Then the elements $\tau_{i}^{\prime} \tau_{i}^{\prime \prime}$ (ordered lexicographically) give a transversal of $H$ in $G$. Let $A$ be a $H$-module. Then

$$
\mathscr{M}_{H \rightarrow G}(A)=\mathscr{M}_{K \rightarrow G}\left(\mathscr{M}_{H \rightarrow K}(A)\right) .
$$

Proposition 1. Let $G>K>H$ be as above.

$$
\mathscr{N}_{H \rightarrow G}(\alpha)=\mathscr{N}_{K \rightarrow G}\left(\mathscr{N}_{H \rightarrow K}(\alpha)\right) \text {. }
$$

Proof. Let $\Phi_{1}: G \rightarrow \mathscr{S}_{l_{1}} \int K, \Phi_{2}: K \rightarrow \mathscr{S}_{l_{2}} \int H, \Phi: G \rightarrow \mathscr{S}_{l} \int H$ be defined with respect to the above transversals. We have a commutative diagram 


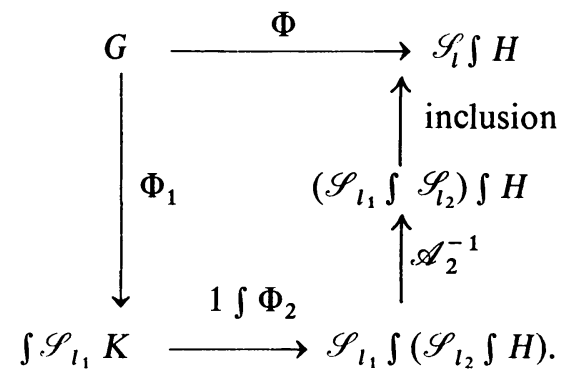

The proposition now follows by applying rules 5-1 and 5-2.

2. Double coset rule. Let $G$ be a group and $H$ and $K$ subgroups of finite index. Let $G=\bigcup_{i=1}^{d} K \tau_{i} H$ be a decomposition of $G$ into disjoint double cosets. Let $K \tau_{i} H=\bigcup_{j=1}^{l_{i}} \rho_{i j} \tau_{i} H$ be a decomposition into disjoint left cosets where $\rho_{i 1}, \rho_{i 2}, \cdots, \rho_{i l_{i}}$ is a left transversal of $\tau_{i} H \tau_{i}^{-1} \cap K$ in $K$. (For convenience write $\tau_{i} H \tau_{i}^{-1}=H_{i}$.) Order the elements $\rho_{i j} \tau_{i}$ lexicographically and define $\Phi: G \rightarrow \mathscr{S}_{l} \int H$ with respect to this transversal of $H$ in $G$. Also define $\Phi_{i}: K \rightarrow \mathscr{S}_{l_{i}} \int H_{i} \cap K$ with respect to the appropriate transversal. Let $c_{\tau}: \tau H \tau^{-1} \rightarrow H$ be the map $\tau \sigma \tau^{-1} \rightarrow \sigma$.

If $A$ is an $H$-module, then it may be viewed as an $H_{i}$-module $A_{i}$ through $c_{i}$ and as an $H_{i} \cap K$-module by restriction. Then as $K$-modules

$$
\mathscr{M}_{H \rightarrow G}(A)=\mathscr{M}_{H_{1} \cap K \rightarrow K}\left(A_{1}\right) \otimes \mathscr{M}_{H_{2} \cap K \rightarrow K}\left(A_{2}\right) \otimes \cdots \otimes \mathscr{M}_{H_{d} \cap K \rightarrow K}\left(A_{d}\right)
$$

where $K$ acts diagonally on the right.

Proposition 2. With notation as above,

$$
\operatorname{res}_{G \rightarrow K} \mathscr{N}_{H \rightarrow G}(\alpha)=\prod_{i=1}^{d} \mathscr{N}_{H_{i} \cap K \rightarrow K}\left(\operatorname{res}_{H_{i} \rightarrow H_{i} \cap K} c_{\tau_{i}}^{* *}(\alpha)\right)
$$

Proof. We have a commutative diagram

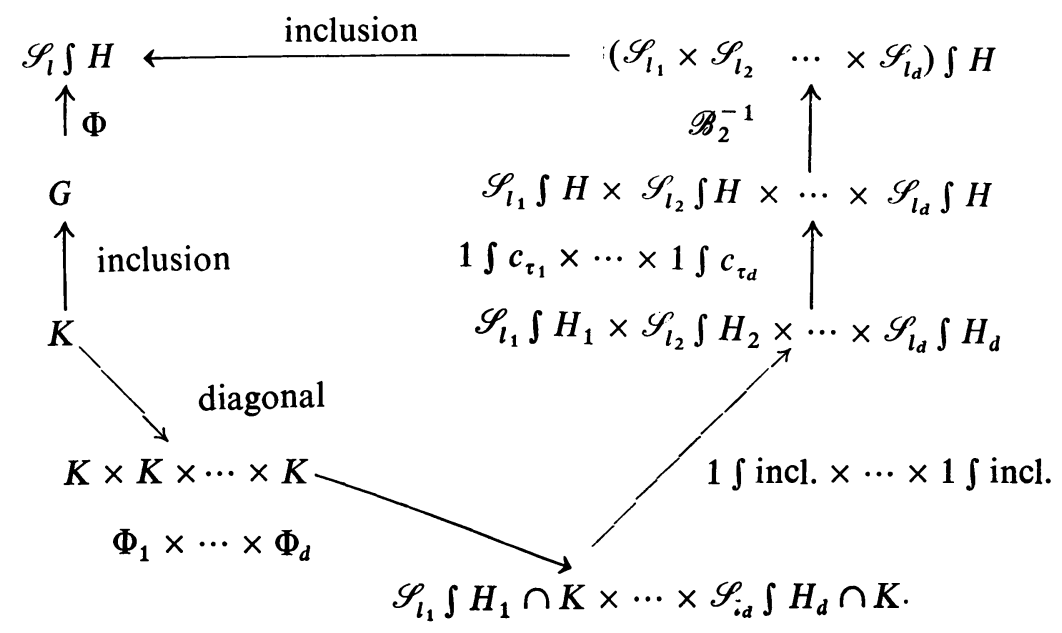


The result follows by applying rules 5-1 and 5-3 and the definition of cup product in the cohomology of $K$.

3. Automorphism property. Let $\phi: G^{\prime} \rightarrow G$ be a homomorphism of groups; let $H$ be a subgroup of finite index $l$ in $G$ and let $H^{\prime}=\phi^{-}(H)$. Suppose $H^{\prime}$ is also of index $l$ in $G^{\prime}$. If $A, A^{\prime}$ are $H, H^{\prime}$ modules respectively and $f: A \rightarrow A^{\prime}$ is a homomorphism consistent with $\phi_{0}=\phi \mid H$, then $f$ induces a homomorphism

$$
\mathscr{M}(f): \mathscr{M}_{H \rightarrow G}(A) \rightarrow \mathscr{M}_{H^{\prime} \rightarrow G^{\prime}}\left(A^{\prime}\right)
$$

consistent with $\phi$.

Proposition 3. Let $\phi: G^{\prime} \rightarrow G$ be as above with $(G: H)=\left(G^{\prime}: H^{\prime}\right)=l$ (where $\left.H^{\prime}=\phi^{-1}(H)\right)$. Then

$$
\phi^{* *}\left(\mathscr{N}_{H \rightarrow G}(\alpha)\right)=\mathscr{N}_{H^{\prime} \rightarrow G^{\prime}}\left(\phi_{0}^{* *}(\alpha)\right) .
$$

Proof. Let $\tau_{1}^{\prime}, \tau_{2}^{\prime}, \cdots, \tau_{l}^{\prime}$ be a transversal of $H^{\prime}$ in $G^{\prime}$; then $\tau_{1}=\phi\left(\tau_{1}^{\prime}\right), \cdots$, $\tau_{l}=\phi\left(\tau_{l}^{\prime}\right)$ is a transversal of $H$ in $G$. Define $\Phi^{\prime}$ and $\Phi$ with respect to these transversals. Consider the commutative diagram

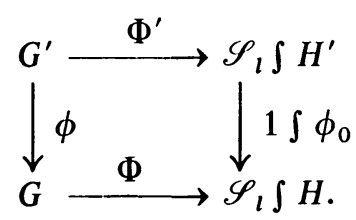

The result follows from $5-1$.

4. Multiplicative property. Let $H$ be a subgroup of $G$ of finite index. If $A_{1} \otimes A_{2} \rightarrow A_{3}$ is a pairing of $H$-modules it induces a pairing of $G$-modules

$$
\mathscr{M}_{H \rightarrow G}\left(A_{1}\right) \otimes \mathscr{M}_{H \rightarrow G}\left(A_{2}\right) \rightarrow \mathscr{M}_{H \rightarrow G}\left(A_{3}\right) .
$$

Proposition 4. With $G>H$ as above,

$$
\mathscr{N}_{H \rightarrow G}(\alpha \cup \beta)=\mathscr{N}_{H \rightarrow G}(\alpha) \cup \mathscr{N}_{H \rightarrow G}(\beta) .
$$

Proof. Let $\Phi: G \rightarrow \mathscr{S}_{l} \int H$ be defined with respect to a transversal. Since any homomorphism of groups induces a multiplicative homomorphism of cohomology, the result follows by applying $\Phi^{* *}$ to the formula (2) of 5-4.

RemarK 1. Since $\mathscr{N}_{H \rightarrow G}$ is multiplicative it defines a homomorphism $\mathscr{N}: U_{e}(H) \rightarrow U_{e}(G)$ from the group of units $U_{e}(H)$ of the ring $H_{e}^{* *}(H, Z)$ to the corresponding group for $G$.

REMARK $2\left({ }^{4}\right)$. It is natural to investigate the quantity $\mathscr{N}_{H \rightarrow G}\left(\operatorname{res}_{G \rightarrow H^{\alpha}} \alpha\right)$ where for example $\alpha \in H^{2 n}(G, Z)$. If the analogy with transfer held true then this quantity would equal $\alpha^{l}$. In fact except in special cases this equality is false.

(4) I should like to thank R. G. Swan for pointing out the substance of this remark to me and also for much valuable advice concerning the contents of this paper. 
For example let $G=P \times H$ where $P$ is cyclic of prime order. Let $\delta: H \rightarrow H^{P}$ be the diagonal homomorphism; then the map $\Phi: G \rightarrow P \int H$ may be chosen to be the obvious induced homomorphism $P \times H \rightarrow P \cdot H^{P}$. Let $X$ be an $H$-projective resolution of $Z$ and $\Delta: X \rightarrow X^{P}$ a diagonal map over $\delta$. If it were possible to choose $\Delta$ to be a P-homomorphism the questioned equality would hold. Unfortunately we know that this is generally not possible, and this fact is responsible for the existence of the Steenrod operations. In fact one can convince oneself that all the Steenrod reduced powers except $u \rightarrow u^{P}$ would be trivial were the equality in question to hold. (Using the properties of the Steenrod operations, we can show that for $\lambda \in H^{2}\left(H, Z_{P}\right), \mathcal{N}(\lambda) \in H^{2 P}\left(P \times H, Z_{P}\right)$ is given by

$$
1 \times \lambda^{P}-\mu^{P-1} \times \lambda
$$

where $\mu$ generates $H^{2}\left(P, Z_{P}\right)$. It is possible also to derive this formula by means of Chern classes.)

7. Applications. Let $G$ be a group and $H$ a subgroup of finite index. Restrict attention to commutative $G$-rings $k$. Notice that this is no essential restriction since if $A$ is a $G$-module, $k=Z \oplus A$ can be made a commutative $G$-ring in which $A^{2}=0$ in the usual way.

THEOREM 1. Let $G$ be a group, $H$ a subgroup of finite index $l$ and $k$ a commutative G-ring. For $\chi \in H^{2}(H, k)$ we have

$$
\mathscr{N}_{H \rightarrow G}(1+\chi)=\mathscr{N}_{0}(\chi)+\mathscr{N}_{1}(\chi)+\cdots+\mathscr{N}_{l}(\chi)
$$

$\left(\mathscr{N}_{i}(\chi) \in H^{2 i}(G, k)\right)$ where $\mathscr{N}_{0}(\chi)=1, \mathscr{N}_{1}(\chi)=\operatorname{tr}_{H \rightarrow G}(\chi)$, and $\mathscr{N}_{l}(\chi)=\mathscr{N}_{H \rightarrow G}(\chi)$.

Proof. Let $\Phi: G \rightarrow \mathscr{S}_{l} \int H$ be defined with respect to some transversal. If $W$ is an $\mathscr{S}_{l}$-resolution and $X$ an $H$-resolution of $Z$, then $W \otimes X^{l}$ is an $\mathscr{S}_{l} \int H$ resolution and through $\Phi$ a $G$-projective resolution of $Z$. Let $\mu: X_{0} \rightarrow Z, \varepsilon: W_{0} \rightarrow Z$ be the augmentations for these resolutions. Suppose the transversal defining $\Phi$ has been chosen so that $\tau_{1}=1$. Then

$$
F=\varepsilon \otimes 1_{X} \otimes \mu^{l-1}: W \otimes X \otimes X^{l-1} \rightarrow X
$$

is a homomorphism of $\mathrm{H}$-projective resolutions of $\mathrm{Z}$ and induces the identity in cohomology for $H$.

Let $\eta: Z \rightarrow k$ be defined by $\eta(1)=1_{k}$. Then $\mu^{\prime}: X_{0} \rightarrow k$ defined by $\mu^{\prime}=\eta \circ \mu$ represents $1 \in H^{0}(H, k)$. Let $f: X_{2 r} \rightarrow k$ represent $\chi \in H^{2 r}(H, k)$. Then in view of 5-5 (and the definition of the map $\left.\psi: k^{l} \rightarrow k\right) \mathscr{N}(1+\chi)$ is represented by $\mathscr{N}\left(\mu^{\prime}+f\right)$ where

$$
\mathscr{N}(g)\left(w_{0} \otimes x_{1} \otimes x_{2} \otimes \cdots \otimes x_{l}\right)=\varepsilon^{\prime}\left(w_{0}\right)\left(\tau_{1} g\left(x_{1}\right)\right)\left(\tau_{2} g\left(x_{2}\right)\right) \cdots\left(\tau_{l} g\left(x_{l}\right)\right) .
$$

The only statement in Theorem 1 which needs more discussion is that concerning transfer. $\mathscr{N}_{1}(\chi)$ is represented by $\mathscr{N}_{1}(f)$ where 


$$
\begin{aligned}
\mathscr{N}_{1}(f)\left(w_{0} \otimes x_{1} \otimes\right. & \left.x_{2} \otimes \cdots \otimes x_{l}\right) \\
& =\sum_{i=1}^{l} \varepsilon^{\prime}\left(w_{0}\right) \mu^{\prime}\left(x_{1}\right) \mu^{\prime}\left(x_{2}\right) \cdots\left(\tau_{i} f\left(x_{i}\right)\right) \cdots \mu^{\prime}\left(x_{l}\right) \\
& =\sum_{i=1}^{l} \tau_{i} \tilde{f}\left(\tau_{i}^{-1}\left(w_{0} \otimes x_{1} \otimes \cdots \otimes x_{l}\right)\right),
\end{aligned}
$$

or $\mathscr{N}_{1}(f)=\operatorname{tr}_{H \rightarrow G} \tilde{f}$ where

$$
\tilde{f}\left(w_{0} \otimes x_{1} \otimes x_{2} \otimes \cdots \otimes x_{l}\right)=\varepsilon^{\prime}\left(w_{0}\right) f\left(x_{1}\right) \mu^{\prime}\left(x_{2}\right) \cdots \mu^{\prime}\left(x_{l}\right) .
$$

(Remember $\tau_{1}=1$ !) However $F^{*}(f)=\tilde{f}$ so that $\tilde{f}$ represents $\chi$ as required.

THEOREM 2. Let $G$ be a group, $H$ a normal subgroup of finite index; let $k$ be a commutative G-ring. Then for $\alpha \in H_{e}^{* *}(H, k)$,

$$
c_{\tau_{1}}(\alpha) \cdot c_{\tau_{2}}(\alpha) \cdots c_{\tau_{l}}(\alpha) \in \operatorname{Im} \operatorname{res}_{G \rightarrow H}
$$

(where $\tau_{1}, \cdots, \tau_{l}$ is a transversal of $H$ in $G$ ).

Proof. Clear from the double coset rule.

THeOREM 3. Let $G$ be a finite group and $H$ a subgroup of $G$. There is an integer $m \mid(G: 1)$ such that

$$
\text { res : } H^{2 q}(G, Z) \rightarrow H^{2 q}(H, Z)
$$

is nontrivial for $q \equiv 0 \bmod m$.

Proof. It is enough to prove this for $H$ cyclic of prime order. Let $\chi$ generate $H^{2}(H, Z)$. By the double coset rule

$$
\operatorname{res}_{G \rightarrow H} \mathscr{N}_{H \rightarrow G}(1+\chi)=\prod_{i=1}^{d} \mathscr{N}_{H_{i} \cap H \rightarrow H} \operatorname{res}_{H_{i} \rightarrow H_{i} \cap H}\left(1+c_{\tau_{i}} \chi\right)
$$

where $G=\bigcup_{i=1}^{d} H \tau_{i} H$ and $H_{i}=\tau_{i} H \tau_{i}^{-1}$.

However,

$$
H_{i} \cap H= \begin{cases}H & \tau_{i} \in \text { Normalizer of } H=N, \\ \{1\} & \text { otherwise. }\end{cases}
$$

Since $\mathscr{N}_{\{1\} \rightarrow H}(1+0)=1$, we can ignore all terms in the product where $\tau_{i} \notin N$. Moreover, in $N$, double cosets are all single cosets. Hence

$$
\operatorname{res}_{G \rightarrow H} \mathscr{N}_{H \rightarrow G}(1+\chi)=\left(1+c_{\tau_{1}} \chi\right)\left(1+c_{\tau_{2}} \chi\right) \cdots\left(1+c_{\tau_{m}} \chi\right)
$$

where $m=(N: H) \mid(G: 1)$. Since the term $c_{\tau_{1}} \chi \cdots c_{\tau_{m}} \chi \neq 0$, Theorem 3 follows. 


\section{BIBLIOGRAPHY}

1. M. F. Atiyah, Charasters and the cohomology of finite groups, Inst. Hautes Études Sci. Publ. Math. No. 9 (1961), 23-64.

2. A. Borel and F. Hirzebruch, Characteristic classes and homogeneous spaces. I, Amer. J. Math. 80 (1958), 458-538, p. 475.

3. H. Cartan and S. Eilenberg, Homological algebra, Princeton Univ. Press, Princeton, N. J., 1956.

4. L. Evens, The cohomology ring of a finite group, Trans. Amer. Math. Soc. 101 (1961), 224-239.

5. P. Hall, The theory of groups, Macmillan, New York, 1959.

6. M. Nakaoka, Homology of the infinite symmetric group, Ann. of Math. (2) 73 (1961), 229-257.

7. R. G. Swan, The nontriviality of the restriction map in the cohomology of groups, Proc. Amer. Math. Soc. 11 (1960), 885-887.

UNIVERSITY OF CALIFORNIA,

BERKeley, CALIFORNia 